TITLE:

\title{
Sampling and Analysis of Animal Scent Signals
}

\author{
AUTHORS AND AFFILIATIONS: \\ David Walker ${ }^{1, *}$, Stefano Vaglio ${ }^{1,2, *}$ \\ ${ }^{1}$ Department of Biology, Chemistry and Forensic Science, University of Wolverhampton, \\ Wolverhampton, UK \\ ${ }^{2}$ Department of Anthropology \& Behavior, Ecology and Evolution Research Centre, Durham \\ University, Durham, UK \\ *These authors contributed equally. \\ Corresponding Author: \\ Stefano Vaglio （S.Vaglio@wlv.ac.uk) \\ Email Addresses of Co-author: \\ David Walker (D.Walker7@wlv.ac.uk) \\ KEYWORDS: \\ headspace solid-phase microextraction, gas chromatography-mass spectrometry, animal \\ communication, scent-marking, olfaction, signalling
}

\section{SUMMARY:}

We have developed an effective methodology for sampling and analysis of odor signals in order to understand how they may be used in animal communication. Particularly, we use headspace solid-phase microextraction coupled with gas chromatography-mass spectrometry to analyze the volatile components of animal odors and scent-markings.

\footnotetext{
ABSTRACT:

We have developed an effective methodology for sampling and analysis of odor signals, by using headspace solid-phase microextraction coupled with gas chromatography-mass spectrometry, to understand how they may be used in animal communication. This technique allows the semi-quantitative analysis of the volatile components of odor secretions by enabling the separation and tentative identification of the components in the sample, followed by the analysis of peak area ratios to look for trends that could signify compounds that may be involved in signaling. The key strengths of this current approach are the range of sample types that can be analyzed; the lack of need for any complex sample preparation or extractions; the ability to separate and analyze the components of a mixture; the identification of the components detected; and the capability to provide semi-quantitative and potentially quantitative information on the components detected. The main limitation to the methodology relates to the samples themselves. Since the components of specific interest are volatile, and these could easily be lost, or their concentrations altered, it is important that the samples are
} 
stored and transported appropriately after their collection. This also means that sample storage and transport conditions are relatively costly. This method can be applied to a variety of samples (including urine, feces, hair and scent-gland odor secretions). These odors consist of complex mixtures, occurring in a range of matrices, and thus necessitate the use of techniques to separate the individual components and extract the compounds of biological interest.

\section{INTRODUCTION:}

Very little is known about the chemical changes underpinning the olfactory signals in animals ${ }^{1}$, also because of methodological challenges in recording and quantifying volatile chemical profiles of odors ${ }^{2}$. There are several potential pitfalls when working with highly complex, chemical matrices; these include when sampling and analyzing the odor samples ${ }^{3}$.

At the Rosalind Franklin Science Center, University of Wolverhampton, we are undertaking the analysis of odors and scent-marks to understand how they may be used by animals. We combine semiochemistry with behavioral ecology, endocrinology and cytology to improve our understanding of the role played by olfactory signals in animal communication.

We have developed a methodology and then analyzed odors and markings from a variety of species including several non-human primates (i.e., crowned lemurs, red-ruffed lemurs, Japanese macaques, olive baboons, chimpanzees) and other mammals (i.e., cats, cows). We have collected and analyzed a variety of samples, including urine, feces, hair and scent-gland odor secretions. These odors and scent-marks consist of complex mixtures of compounds and therefore any methodology used for their analysis need to include some form of separatory technique. As illustrated, they also occur in a range of matrices which necessitates the use of techniques to extract the components of interest.

Previous studies by Vaglio et al. ${ }^{4}$ and other authors ${ }^{5}$ used dynamic headspace extraction (DHS) with gas chromatography-mass spectrometry (GC-MS) while direct solvent extraction ${ }^{6}$ and complex solvent extractions ${ }^{7}$ have also been used. Particularly, dynamic headspace sampling involves purging the headspace with a known volume of inert gas that ultimately removes all volatile compounds with the exception of those showing a strong affinity for the sample matrix (for example, polar compounds in aqueous samples).

For the current methodology, we have adopted the technique of headspace solid-phase microextraction (HS-SPME) coupled with GC-MS. In particular, we have developed and enhanced the methodology already used by Vaglio et al. in its previous GC-MS laboratory ${ }^{8-10}$.

Solventless extraction techniques are very effective for analyzing small, highly volatile compounds (which otherwise can be lost easily from a sample) because these methods immobilize compounds on a stable, solid phase support. The HS-SPME uses a fiber coated with an adsorbent polymer to capture volatile compounds in the sample headspace or to extract dissolved compounds by immersion in an aqueous biological fluid ${ }^{11}$. The polymer coating does not bind the compounds strongly, therefore by heating in the injection port of the GC they can be removed. This method is more powerful than solvent extraction techniques and also more 
effective than DHS.

In the current approach samples are contained within glass vials. These vials are warmed to a temperature of $40{ }^{\circ} \mathrm{C}$ to simulate animal body temperature in order to promote the volatile components of the scent-mark to occupy the head-space of the vial. A SPME fiber, coated with $65 \mu \mathrm{m}$ of polydimethylsiloxane/divinylbenzene (PDMS/DVB) sorbent material, is exposed to the headspace environment and volatile components from the sample are adsorbed onto the fiber. On heating the fiber in the inlet port of a GC-MS, the volatile components are desorbed from the fiber and then separated by the GC. Mass spectral fragmentation patterns are obtained for each component using the MS. By comparison of these mass spectra against mass spectral databases, it can be possible to tentatively identify the components of the scent-mark. Through the use of an auto-sampler, we are able to analyze multiple samples in batches in a consistent manner.

Given that each type of SPME fiber has a different affinity with polar chemicals, the fiber is usually chosen depending on the polarity and/or molecular weight of the target chemical compounds. In addition, the GC conditions are changed depending on the type of GC column and the characteristics of the target chemical compounds.

This technique allows the semi-quantitative analysis of the volatile components of scentmarkings by enabling the separation and tentative identification of the components in the sample, followed by the analysis of peak area ratios to look for trends that could signify components of the scent-marking that may be involved in signaling.

The key strengths of this current approach are:

- The range of sample types that can be analyzed.

- No complex sample preparation or extractions are required.

- The ability to analyze volatile components.

- The ability to separate the components of a mixture.

- To be able to identify the components detected.

- The ability to provide semi-quantitative and potentially quantitative information on the components detected.

\section{PROTOCOL:}

\section{Sample collection}

\subsection{Sample odors that are one of the following:}

1.1.1. Collect spontaneously released by habituated study subjects (e.g., zoo primates) via scent-marking on sterile filter paper (e.g., scent-gland odor secretions) or directly into vials (e.g., urine). 
1.1.2. Collect by rubbing sterile cotton swabs after training study subjects by using positive reinforcement training.

1.1.3. Collect by rubbing sterile cotton swabs after sedation of study subjects.

1.2. Place samples into sterile $10 \mathrm{~mL}$ screw-capped clear glass vials, and seal with screw topped caps incorporating FTFE/silicone septa. Immediately store them at $-20^{\circ} \mathrm{C}$.

NOTE: It is vital to use clean personal protective equipment, such as nitrile gloves; change them frequently; avoid direct skin contact with samples and vials. It is preferred to use brand-new vials; however, in case of used vials, it is vital to pre-clean the vials, and then use the same protocol.

1.3. Take environmental blanks every time scent-marks are collected. For example, collect the sampling media (e.g., filter paper or swab) and a headspace vial that are exposed to the environment while sampling is undertaken.

\section{Sample preparation}

2.1. Prepare samples in the field by cutting with a blade an approximate $10 \mathrm{~mm}$ square from a scent-marked filter paper, or the head of the swab, and placing it in a $10 \mathrm{~mL}$ screw-topped head-space vial.

2.2. After each sample has been prepared, dispose or clean the blade used to cut the sampling media using an appropriate antibacterial wipe and/or alcohol and dry thoroughly.

2.3. Store all samples at $-20^{\circ} \mathrm{C}$.

NOTE: Preferred at $-20^{\circ} \mathrm{C}$ or otherwise as low as practically possible in the field.

\section{Preparation for analysis}

3.1. Remove samples from the freezer and allow to warm naturally to room temperature for at least $1 \mathrm{~h}$.

3.2. Set up the analytical method on the GC-MS as follows:

3.2.1. For SPME analysis conditions, follow the manufacturer's directions to condition SPME fibers before first use: fiber pre-condition $\left(260{ }^{\circ} \mathrm{C}\right.$ for 5 $\min )$, sample incubation $\left(40{ }^{\circ} \mathrm{C}\right.$ for $2 \mathrm{~min}$ ), extraction time (15 $\left.\mathrm{min}\right)$, desorption time $(2 \mathrm{~min})$, and fiber post-condition $\left(260^{\circ} \mathrm{C}\right.$ for $20 \mathrm{~min}$ ). 
3.2.2. Use the following GC conditions: column (HP5-MS $30 \mathrm{~m} \times 0.25 \mathrm{~mm} ; 0.25$ $\mu \mathrm{m})$, injector temperature $\left(270{ }^{\circ} \mathrm{C}\right)$, flow rate $(1 \mathrm{~mL} / \mathrm{min})$, injection mode (splitless), GC oven profile $\left(45^{\circ} \mathrm{C}\right.$ for $2 \mathrm{~min} ; 4^{\circ} \mathrm{C} / \mathrm{min}$ to $170{ }^{\circ} \mathrm{C} ; 20^{\circ} \mathrm{C} / \mathrm{min}$ to $\left.300^{\circ} \mathrm{C}\right)$, MSD transfer line $\left(280^{\circ} \mathrm{C}\right)$.

NOTE: To improve between sample retention time consistency the analytical method is retention time locked).

3.2.3. Use the following MSD conditions: solvent delay $(2.5 \mathrm{~min})$ and scan range (29 to $400 \mathrm{amu}$ ).

NOTE: A range of 10 to 400 was used in previous protocols ${ }^{4}$.

3.3. Ensure that the purge gas supply to the fiber conditioning unit is turned on.

NOTE: It is vital that the SPME assembly is correctly installed in the auto-sampler and that it is aligned to the auto-sampler trays, fiber conditioning unit and GC inlet port. Incorrect alignment could result in damage or destruction of the SPME fiber.

\section{Analysis}

4.1. Place an empty headspace vial (to act as a system blank) in the first position of the GC-MS auto-sampler tray. Place the environmental blank in the second position of the auto-sampler tray. Place the samples for analysis in the subsequent positions of the auto-sample tray.

4.2. Create an analytical sequence to analyze each sample within the sample tray.

4.2.1. In the MassHunter home screen, select Sequence | Load sequence.

4.2.2. Complete the sequence table for all blanks and samples by inserting the appropriate information. Save the completed sequence table.

NOTE: The exact information for the sequence table will be dependent on the laboratories formatting for the table. Minimum information would normally include sample type, sample name, vial location and number, analytical method and data file location and name (allocation of a data file name that matches the sample name aids future data processing). Additional samples can be added to the sequence during the analysis.

\subsection{Run the sequence by selecting Sequence | Run sequence.}

4.4. After analysis return samples to the freezer as soon as possible. 
NOTE: it may be possible to reanalyze samples, but it should be noted that some volatile components may have been totally extracted during the initial analysis and some compounds may have undergone thermal and bacterial decomposition at $40{ }^{\circ} \mathrm{C}$, thus the resulting chromatogram may not be thoroughly representative of the original scent-marking.

\section{Data analysis}

NOTE: Initial data analysis includes the integration of chromatograms to obtain retention time and peak area data together with tentative identification of peaks using ChemStation software and NIST (National Institute of Standards and Technology) mass spectral databases, version MSD F.01.01.2317. Data analysis can be carried out either manually or through a semiautomated method. If the semi-automated method is used then it is sometimes beneficial to undertake a degree of manual data analysis to verify tentative identifications.

5.1. Open the data file by clicking on the appropriate file in the left-hand navigation bar. The total ion chromatogram (TIC) will be displayed in the top window of the data analysis screen.

5.2. To integrate the TIC using the RTE integrator, select Chromatogram | Integrate.

5.3. Adjust integration parameters so that peaks that are greater than $3 \times$ baseline noise are integrated. Select Chromatogram | MS Signal Integration Parameters. In the output box adjust the minimum peak area as appropriate (1.0 produces acceptable results in our examples).

5.4. To identify peaks and generate a summary report, select Export Reports I Library search results report to XLS.

NOTE: The spectral libraries to be searched together with the number of library matches to be displayed need to be preset within the software before a library search can be undertaken.

5.5. The resulting spreadsheet report contains integration data for each peak and a tentative spectral library match to assign identity. Typically, the library quality/library match should be $>80$ to accept the tentative identification. Save the spreadsheet.

5.6. Identify a peak directly from the TIC.

5.6.1. Choose the peak of interest.

5.6.2. If the peak is small, zoom in by drawing a box around the peak by holding the left hand mouse button down, stretch the box over the peak and release. 
5.6.3. Place the cursor line so that it is at the highest point of the peak (or just after).

5.6.4. Double click the right hand mouse button and the mass spectrum for the peak will appear in the lower window of the data analysis screen.

5.6.5. To search the spectral library, move the cursor anywhere in the spectral window and double click the right hand mouse button. The library search results will appear in a new window.

5.6.6. To remove the background noise from a spectrum of interest, first double click the right hand mouse button on the peak in question. Then double click the right hand mouse button in an area with no peaks immediately in front of the peak of interest. Select Chromatogram | Subtract spectra. The subtracted spectrum will display in the lower window of the data analysis screen and will display '(-)' next to the SCAN data in the window header.

\section{REPRESENTATIVE RESULTS:}

Following this protocol, we tentatively identified a total of 32 volatile chemical compounds from the analysis of 14 ano-genital scent-marks spontaneously released on filter paper by redruffed lemurs (Varecia variegata rubra) and compared odor profiles with features of the signaler ${ }^{12}$. Naturally occurring volatile compounds, such as hydrocarbons, terpenes, terpene alcohols and ketones, were present within these profiles and included compounds that had previously been found to act as sex pheromones and cues to fitness in other animal species. The compounds that have been tentatively identified are listed in Table 1. Representative chromatograms ( 1 from a control and 1 from a lemur scent-mark) are shown in Figure 1 . The number and relative abundance of the components varied from sample to sample across different study subjects. However, six compounds (benzaldehyde, 2-ethyl-1-hexanol, p-cresol, cis-p-mentha-2,8-dien-1-ol, 2-pinen-4-one, pentadecane) were present in all samples.

The results of this study suggested that red-ruffed lemurs use scent-marking to convey information about sex and female age, with ano-genital marking playing a role in socio-sexual communication.

Another representative outcome following the use of this protocol was our study of fertility advertisement by female olive baboons (Papio anubis) (Vaglio et al. unpublished data). We identified a total of 74 volatile compounds from the analysis of 385 female baboon vaginal odor samples. These compounds included a range of naturally occurring odorous volatile compounds such as ketones, alcohols, aldehydes, terpenes, volatile fatty acids and hydrocarbons. Typical chromatograms used to compare blank control and female baboon vaginal odor samples from fertile and non-fertile periods are shown in Figure 2. We examined the relationships between vaginal odor profiles and sexual receptivity of female baboons. Our results showed that the total amount of vaginal odor differs with fertility suggesting that odor might play a role in 
signaling female baboon fertility. We also found differences in vaginal odor between group types but we could not distinguish the effects of group composition, female age and parity.

\section{FIGURE AND TABLE LEGENDS:}

Figure 1. Example chromatograms; (top chromatogram - 'control') the control sample, showing contaminants; (bottom chromatogram - 'lemur scent-mark') one adult female red-ruffed lemur ano-genital odor secretions, showing contaminants and meaningful biological compounds. Red arrows indicate the six meaningful biological compounds which were found in all samples: (a) benzaldehyde; (b) 2-ethyl-1hexanol; (c) p-cresol; (d) cis-p-mentha-2,8-dien-1-ol; (e) 2-pinen-4one; ( $f$ ) pentadecane.This figure has been modified from Janda et al. ${ }^{12}$.

Figure 2. Example chromatogram from (top chromatogram - 'control') control sample, showing contaminants; (middle chromatogram - 'baboon non-fertile odor') female olive baboon, vaginal odor sample from non-fertile period; and (bottom chromatogram - 'baboon fertile odor') female olive baboon, vaginal odor sample from fertile period.

Table 1. Volatile compounds present in filter paper samples from female red-ruffed lemur anogenital odor secretions identified tentatively using ChemStation software and NIST mass spectral databases, version MSD F.01.01.2317. This table has been modified from Janda et al. ${ }^{12}$.

\section{DISCUSSION:}

The use of control samples, both environmental controls created at the time of sample collection and system blanks, are crucial for the interpretation of the scent-mark samples. Any peaks attributed to the sampling environment or the instrumental system need to be excluded from scent-mark samples so that only the peaks of interest are included in any interpretation. These controls can also play a role in assessing and monitoring the 'health' of the instrumentation.

The protocol includes steps to condition the fiber before and after each extraction. This is made easier by the use of an auto-sampler and ensures that there is no cross contamination from sample to sample.

The main limitation to the methodology relates to the samples themselves. Following collection, it is important that they are stored and transported appropriately. The components of specific interest are volatile, and these could easily be lost, or their concentrations altered. Currently samples are stored and transported frozen, typically at $-20^{\circ} \mathrm{C}$. As a result there is a significant cost involved in the storage and transport of these samples. Delays in transporting samples to the laboratory for analysis will increase these costs further and could potentially affect the results obtained from the scent-marks. Further research is needed to understand the effect of time and storage on the analytical results obtained from samples. As the components of particular significance are the more volatile components of the scent-marks, it is possible that the levels of these components may alter post sample collection. Consideration may also 
need to be given to potential bacterial effects within some sample types. For example, in alcohol testing of urine samples, bacteria present in the urine may produce alcohol and therefore increase the levels of alcohol detected.

The current methodology meets all of the requirements previously outlined. It provides good quality results from which further information on the composition of scent-marks is being obtained. However, the analysis is a laboratory based technique that is dependent on the samples being submitted to the laboratory. One potential solution to this limitation would be through the use of portable GC-MS instrumentation, instruments that could be taken into the field where the samples are being taken. This approach would alleviate the need to store and transport samples and would enable the real time analysis of scent-marks potentially providing greater information on the most volatile components of the marks. Several portable GC-MS instruments are available. They utilize different technology to that found in the laboratory based instrumentation, but should provide comparable results. The use of the HS-SPME extraction technique is still applicable. However, as a result of being portable, the instruments do not offer the option of automated sample introduction; nevertheless, if samples are analyzed as soon as they are collected, daily sample numbers are likely to be manageable for manual injection. Also, in case of field extraction, care should be taken that the SPME fiber does not trap environmental chemicals before use. Currently available instruments are battery powered enabling total portability, but with the provision of some form of power supply (even a small generator), these instruments could be operated for longer periods of time. These instruments by their nature are often designed to be simple to operate, with minimal training and knowledge required. This means that it may not be necessary for highly trained operators to be deployed with the instrument and that they could be operated by those collecting the samples. Detailed interpretation of analytical results could be achieved remotely or when the instrument returns to base.

Another major limitation with methods using SPME fibers is that it is possible to analyze only chemical compounds that exist in abundance in the samples. In particular, far larger amounts of the chemical exist in the samples even when the amount of molecules is subthreshold for the analysis using SPME fibers. In addition, in the context of odor secretions released by animal scent-marks and other animal chemical signals, individual animals tend to use chemical compounds in small amounts to communicate with other animals and/or detect olfactory signals from materials. In other words, the different performances of animal noses and SPME extraction may represent a major challenge to the success of this sampling technique.

Future development of the current methodology could be based around sample collection with the investigation into the use of alternative sorbent materials or sorbent tubes for use with thermal desorption systems. Such developments may go some way to help sample storage and transport conditions.

\section{ACKNOWLEDGMENTS:}

We thank Keith Holding for his assistance with chemical analyses at the Rosalind Franklin 
Science Center, Wolverhampton, and Ben Mantle for the production of the video. We are also grateful to Prof. Gloriano Moneti, Dr. Giuseppe Pieraccini and the members of the University of Florence's Mass Spectrometry Center, Florence, and to Prof. Luca Calamai and Dr. Marco Michelozzi of the CNR's ARCA Lab, Florence, for their help with setting up this methodology. The research projects which included the sampling and analysis methods described in the manuscript were supported by two Marie Skłodowska-Curie Intra European Fellowships (Grant Agreement IDs: 327083, 703611), a small grant ('The sensory enriched primate') from the Primate Society of Great Britain, and a small research grant ('Do hunter-gatherers have a special sense of smell?') from the British Academy/The Leverhulme Trust to S.V. The lab work needed to set up this methodology also received funding from the Faculty of Science and Engineering's Annual Funding Competition (Wolverhampton) to S.V.

\section{DISCLOSURES:}

The authors have nothing to disclose.

\section{REFERENCES:}

1. Wyatt, T. D. Pheromones and Animal Behavior: Chemical Signals and Signatures. Cambridge University Press. Cambridge, UK (2014).

2. Heymann, E. W. The neglected sense-olfaction in primate behavior, ecology, and evolution. American Journal of Primatology. 68 (6), 519-524 (2006).

3. Drea, C. M., Boulet, M., DelBarco-Trillo, J. The "secret" in secretions: Methodological considerations in deciphering primate olfactory communication. American Journal of Primatology. 75 (7), 621-642 (2013).

4. Vaglio, S. et al. Sternal gland scent-marking signals sex, age, rank and group identity in captive mandrills. Chemical Senses. 41(2), 177-186 (2016).

5. Marneweck, C., Jürgens, A., Shrader A. M. Dung odours signal sex, age, territorial and oestrous state in white rhinos. Proceedings of the Royal Society of London B: Biological Sciences. 284 (1846) (2016).

6. Shear, W. A., Jones, T. H., Miras, H. M. A possible phylogenetic signal in milliped chemical defenses. Biochemical Systematics and Ecology. 35, 838-842 (2007).

7. Kimura, R. Volatile substances in feces, urine and urine-marked feces of feral horses. Canadian Journal of Animal Science. 81 (3), 411-420 (2001).

8. Vaglio, S., Minicozzi, P., Bonometti, E., Mello, G., Chiarelli, B. Volatile signals during pregnancy: a possible chemical basis for mother-infant recognition. Journal of Chemical Ecology. 35 (1), 131-139 (2009).

9. Setchell, J. M. et al. Chemical composition of scent-gland secretions in an Old World monkey (Mandrillus sphinx): influence of sex, male status, and individual identity. Chemical Senses. 35 (3), 205-220 (2010).

10. Setchell, J. M. et al. Odour signals MHC genotype in an Old World monkey. Proceedings of the Royal Society of London B: Biological Sciences. 278 (1703), 274-280 (2011).

11. Pawliszyn, J. Solid phase microextraction: theory and practice. Wiley-VCH. New York, US (1997).

12. Janda, E. D., Perry, K., Hankinson, E., Walker, D., Vaglio, S. Sex differences in scent- 
marking in captive red-ruffed lemurs. American Journal of Primatology. 81 (1), e22951 (2019). 
Figure 1.

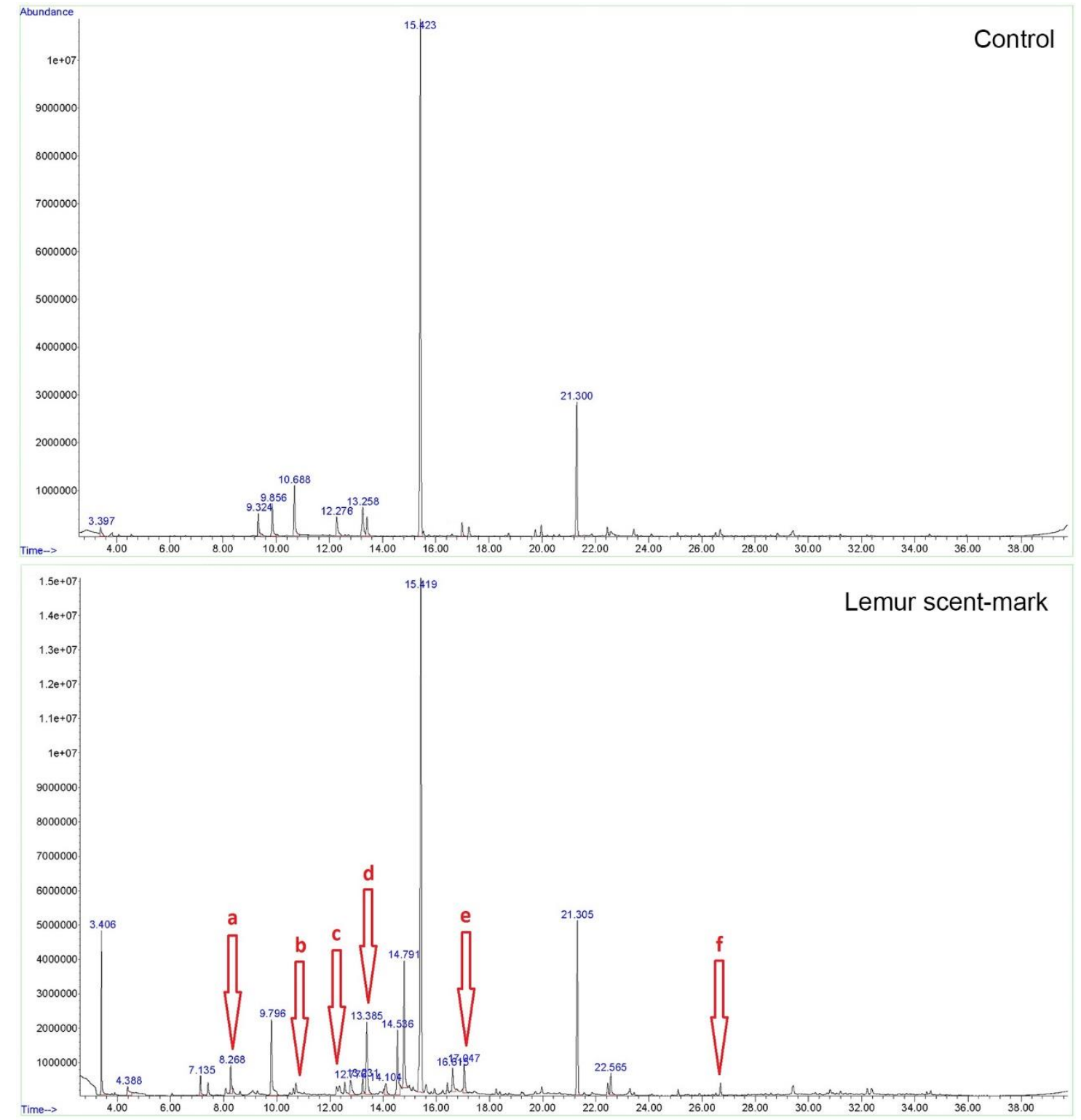


Figure 2.
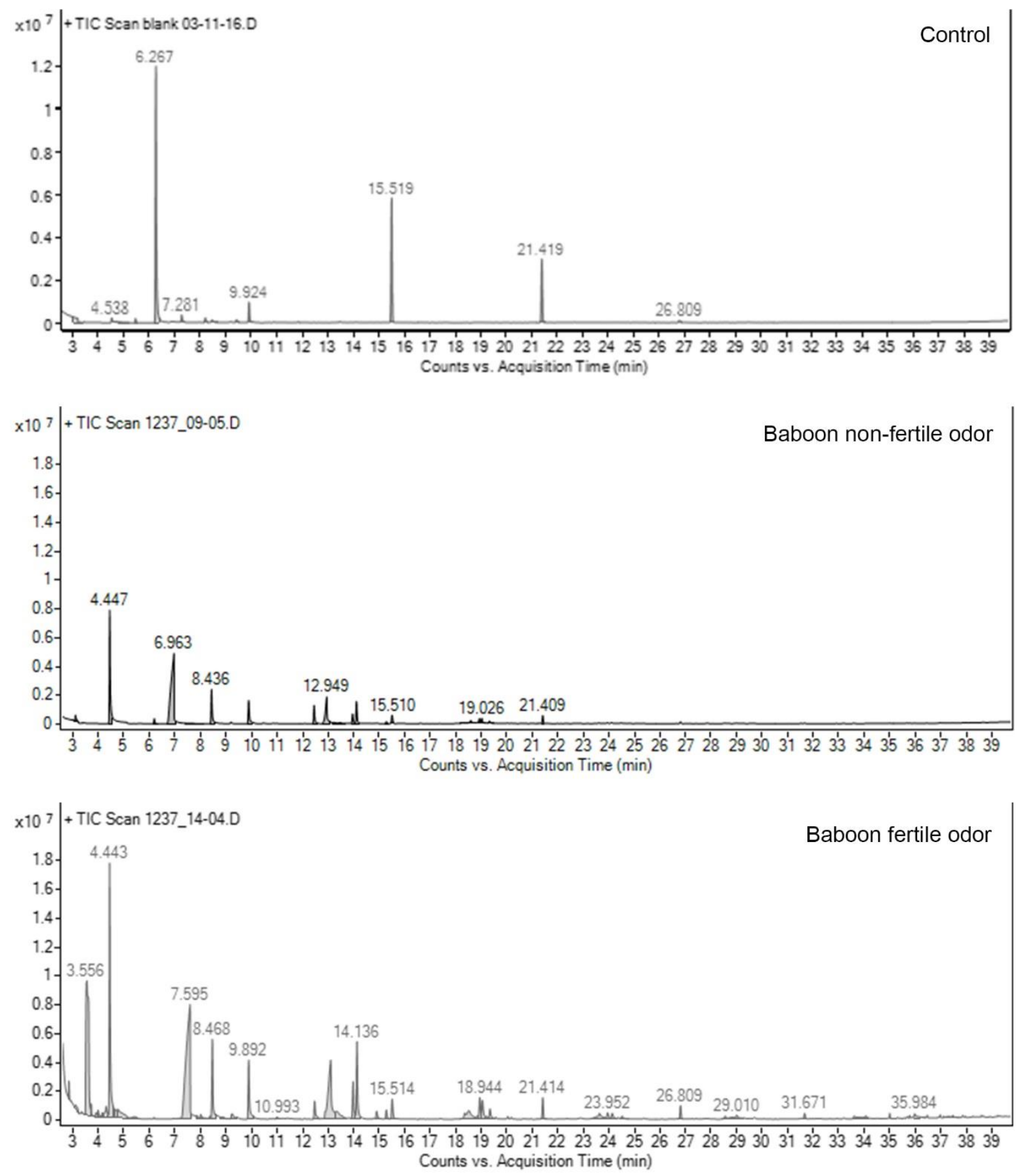
Table 1.

\begin{tabular}{|c|c|c|}
\hline Retention Time (mins) & Tentative Compound ID & Molecular Weight \\
\hline 3.906 & Hexanal & 100 \\
\hline 6.057 & 5-methyl-3-hexanone & 114 \\
\hline 7.413 & Alpha-pinene & 136 \\
\hline 8.077 & 1-isopropyl-4-methylenebicyclo[3.1.0]hex-2-one & 134 \\
\hline 8.268 & Benzaldehyde & 106 \\
\hline 8.623 & 3,7,7-trimethyl-1,3,5-cycloheptatriene & 134 \\
\hline 9.096 & Phenol & 94 \\
\hline 9.269 & 6-methoxy-5-hepten-2-one & 126 \\
\hline 10.72 & 2-ethyl-1-hexanol & 130 \\
\hline 12.362 & p-Cresol & 108 \\
\hline 12.553 & cis-Verbenol & 152 \\
\hline 13.385 & cis-p-Mentha-2,8-dien-1-ol & 152 \\
\hline 14.104 & 1,7,7-Trimethylbicyclo[2.2.1]hepta-2-one & 152 \\
\hline 14.536 & L-Pinocarveol & 152 \\
\hline 14.791 & trans-Verbenol & 152 \\
\hline 15.605 & p-Ethyl-phenol & 122 \\
\hline 15.928 & Terpinen-4-ol & 154 \\
\hline 16.415 & Alpha-Terpineol & 154 \\
\hline 16.615 & Myrtenol & 152 \\
\hline 17.047 & 2-Pinen-4-one & 150 \\
\hline 18.252 & Carvone & 150 \\
\hline 19.217 & p-Mentha-1,8-dien-3-one & 150 \\
\hline 23.283 & 4,7,7-Trimethylbicyclo[4.1.0]hept-3-ene-2-one & 150 \\
\hline 23.443 & Tetradecane & 198 \\
\hline 25.094 & Geranylacetone & 194 \\
\hline 25.899 & Isomethylionone & 206 \\
\hline 26.513 & Pentadecane & 212 \\
\hline 30.871 & 2,6,10-Trimethylpentadecane & 254 \\
\hline 32.208 & Heptadecane & 240 \\
\hline 32.372 & 2,6,10-Trimethylhexadecane & 268 \\
\hline 34.446 & n-Tetracosane & 338 \\
\hline 34.591 & 2,6,10,14-Tetramethylhexadecane & 282 \\
\hline
\end{tabular}

